\title{
Correction to: Identifying Outstanding Transition-Metal-Alloy Heterogeneous Catalysts for the Oxygen Reduction and Evolution Reactions via Subgroup Discovery
}

\author{
Lucas Foppa ${ }^{1,2} \cdot$ Luca M. Ghiringhelli ${ }^{1,2}$ (D)
}

Published online: 5 December 2021

(c) Springer Science+Business Media, LLC, part of Springer Nature 2021

\section{Correction to: Topics in Catalysis https://doi.org/10.1007/s11244-021-01502-4}

The original version of this article unfortunately contained an error and it is corrected with this erratum.

The correct expression of the utility function, defined by Eq. 6, is $u(P, S G)=\frac{\operatorname{std}(P)-s t d(S G)}{\operatorname{std}(P)}$. By using the difference of standard deviations in the utility function (and not their ratio, as indicated in the paper), we favour the selection of SGs that present narrow distributions of values for the target. This correction also concerns Table S1 and Fig. S1 of the ESI, where the expression of the utility function is shown. The code used for the SGD approach uses the correct expression for utility function evaluation. Therefore, the results and discussions are not affected by this correction.

Publisher's Note Springer Nature remains neutral with regard to jurisdictional claims in published maps and institutional affiliations.

The original article can be found online at https://doi.org/10.1007/ s11244-021-01502-4.

Lucas Foppa

foppa@fhi-berlin.mpg.de

1 The NOMAD Laboratory, Fritz-Haber-Institut der MaxPlanck-Gesellschaft, Faradayweg 4-6, 14195 Berlin, Germany

2 Humboldt-Universität zu Berlin, Zum Großen Windkanal 6, 12489 Berlin, Germany 